\title{
Evguenii Konstantinovitch Zavoïskii (1907-1976) et la découverte de la résonance paramagnétique électronique
}

Alexandre Semerok $^{(1)}$ (alexandre.semerok@cea.fr), Edgar Soulié(2) (edgar.soulie@cea.fr) et Natalia E. Zavoïskaya ${ }^{(3)}$

(1) SEARS, DEN, CEA/Saclay, 91191 Gif-sur-Yvette Cedex

(2) Ancien ingénieur-chercheur au Département de physico-chimie du CEA/Saclay (3) Historienne, fille de E.K. Zavoïskii

Evguenii Konstantinovitch

Zavoïskii fut un éminent physicien expérimentateur russe.

En 1944, à Kazan en URSS, il réalisa l'expérience décisive de la découverte de la résonance paramagnétique électronique (RPE).

\section{La RPE permet d'étudier}

la structure électronique de molécules paramagnétiques.

Elle a un impact significatif en physique, chimie, minéralogie, biologie, médecine et physiologie. Avec son talent original d'expérimentateur et sa profonde intuition physique, Zavoïskii a aussi mené des recherches brillantes sur la physique nucléaire, la fusion thermonucléaire contrôlée, l'électronique physique et les phénomènes rapides.

\section{Sa jeunesse et les débuts de sa carrière scientifique}

Evguenii Zavoïskii (fig. 1), né le 28 septembre 1907 dans la ville de MoghilevPodolski (à l'époque dans l'Empire russe, actuellement en Ukraine, à $300 \mathrm{~km}$ au sud-ouest de Kiev), était le troisième des cinq enfants d'un médecin militaire et d'une enseignante d'école publique.

Lorsqu'il n'avait qu'un an, les activités professionnelles de son père incitèrent la famille à déménager à Kazan. Très jeune, Evguenii s'intéresse aux expériences de physique et de chimie. Ses parents (fig. 2) soutiennent et encouragent cet intérêt et cet enthousiasme. En 1919, son père Konstantin Zavoïskii meurt du paludisme à l'âge de 46 ans et, en 1921, à la fin de la guerre civile en Russie, la famille déménage à Slobodskoï (dans la région de Kirov) pour échapper à la famine qui sévit dans le pays ; elle y séjourne cinq ans.

En 1926, après ses études à l'école secondaire, Evguenii passe l'examen d'entrée à l'Université de Kazan. Il participe activement aux associations physicomathématiques de l'Université. À l'instigation du professeur V.A. Ul'yanin (18631931), il est envoyé à Leningrad, où il développe une nouvelle méthode de production d'ondes très courtes. En 1933,

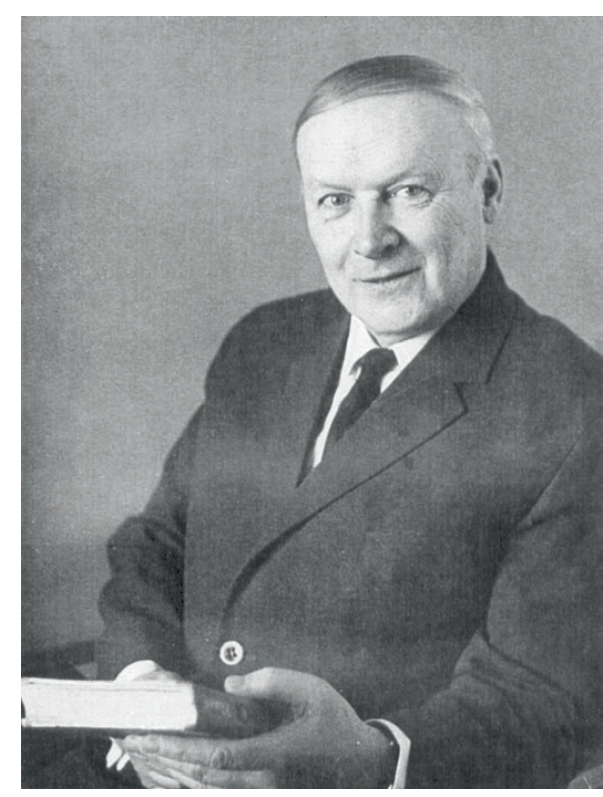

1. Evguenii Konstantinovitch Zavoïskii en 1969.

il soutient sa thèse de doctorat et devient professeur associé et titulaire de la chaire de physique expérimentale de l'Université d'État de Kazan.

Dans les années 1933-1935, ses activités de recherche scientifique sont étroitement liées à la détection et à l'étude de l'interaction des rayonnements de radiofréquence élevée avec la matière. Zavoïskii fait de nombreuses expériences sur l'absorption résonnante de champs électromagnétiques 


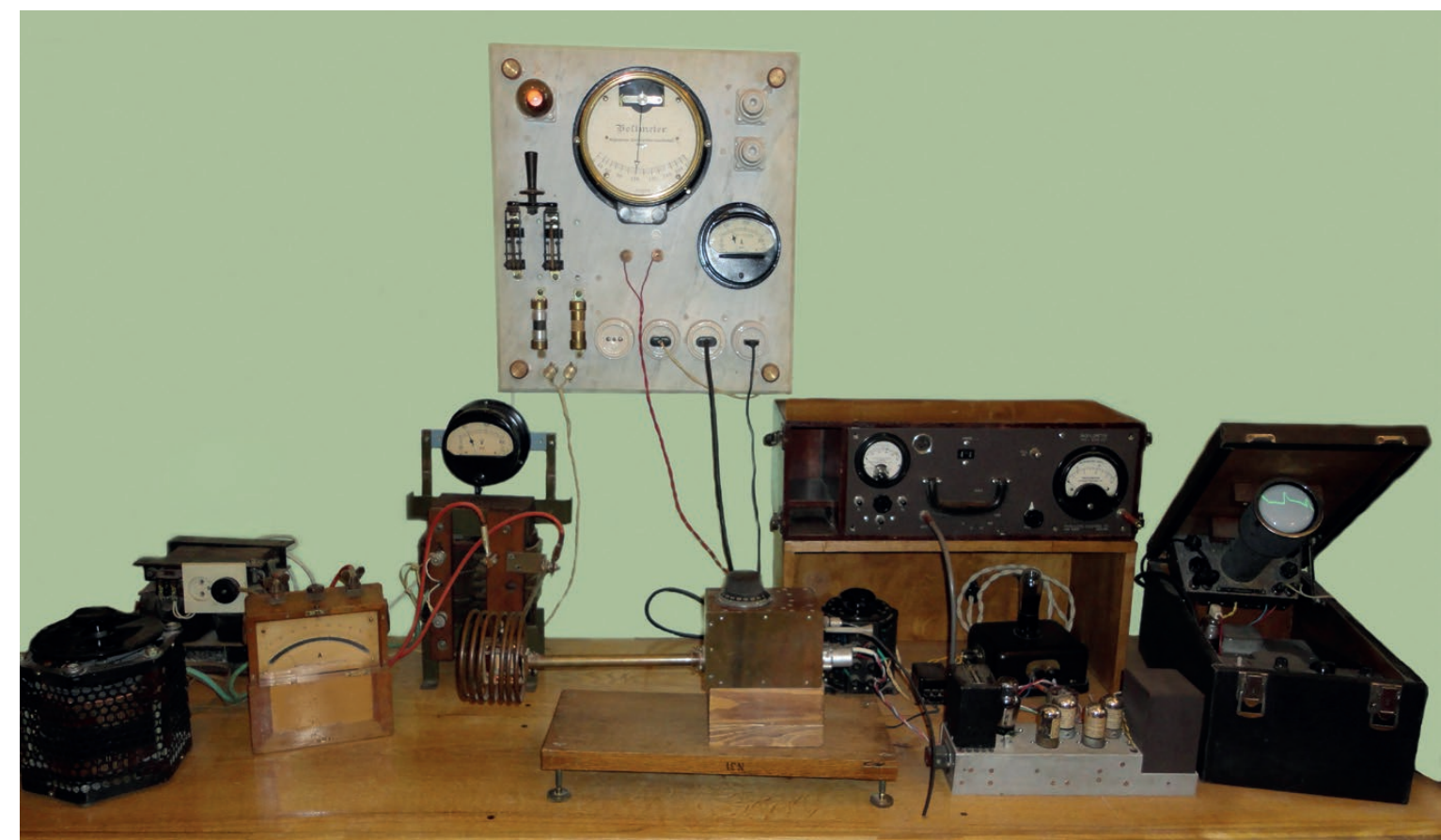

Reconstitution du spectromètre de RPE de Zavoïskii, effectuée par Igor I. Silkin, créateur et directeur du musée Zavoïskii à Kazan.

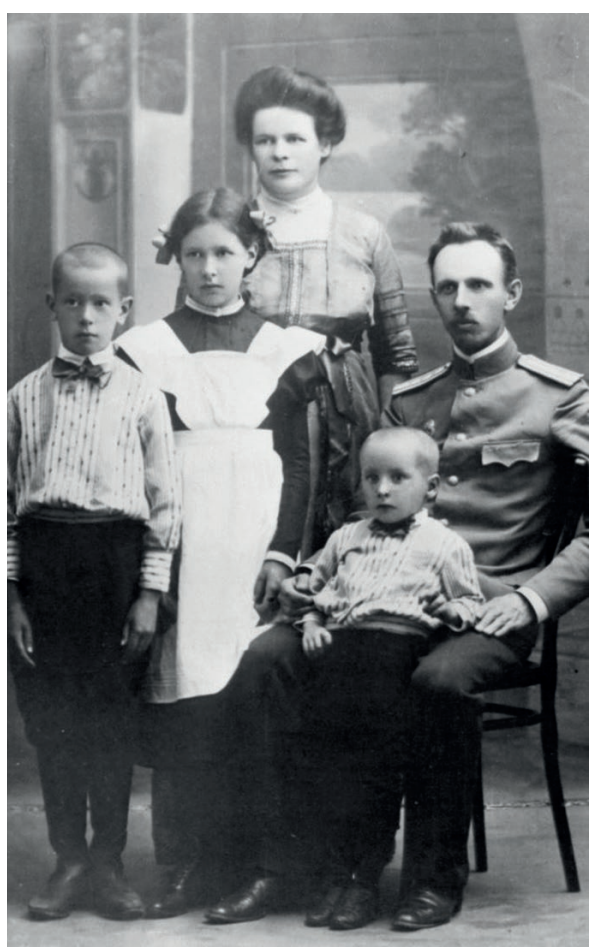

2. Famille de E. K. Zavoïskii en 1912.

De gauche à droite : Boris (1903-1937), Tatiana (1900-1978), Elizaveta Nikolaevna Zavoïskaya (née Egoroushkina-Kalashnikova, mère, 18801941), Evguenii (1907-1976) et Konstantin Ivanovitch Zavoïskii (père, 1873-1919). La quatrième enfant, Vera (1910-1979), est absent et le cinquième, Viatcheslav (1916-2001), n'est pas encore né. radiofréquence par les liquides et les gaz et lance un programme d'études expérimentales sur les effets physiques et chimiques des radiofréquences ultracourtes (de longueur d'onde de l'ordre du mètre) sur la matière. Ses premières tentatives d'observation de la résonance électrique dans le domaine des radiofréquences échouent.

\section{Premières études sur la résonance magnétique nucléaire (RMN)}

En 1936, Zavoïskii suggère une méthode dite du " courant de grille " pour mesurer les potentiels d'excitation des atomes et des molécules [1]. Il présente les bases théoriques prouvant la possibilité d'exciter les molécules avec des électrons accélérés par des champs électriques à haute fréquence. Son modèle théorique a été validé par de nombreux résultats expérimentaux obtenus dans les gaz.

Zavoïskii et son collègue Boris M. Kozyrev confirment l'effet de l'absorption de rayonnement à haute fréquence (environ $56 \mathrm{MHz}$ ) dans les gaz, les liquides et les liquides gelés, et ils signalent l'augmentation du taux de chauffage lorsqu'on applique un champ magnétique constant [2]. Zavoïskii et Kozyrev ont prouvé expérimentalement que cet effet peut témoigner de certains processus intermoléculaires et permet de révéler la structure moléculaire détaillée.

En 1940, Zavoïskii et ses collègues Semen A. Altshuler et Boris M. Kozyrev (fig. 3) montent une expérience destinée à mettre en évidence le phénomène de résonance magnétique des protons en phase condensée. Zavö̈skii s'était inspiré des travaux du Néerlandais C.J. Gorter. Ce dernier avait tenté de déterminer le moment magnétique d'un noyau par la méthode calorimétrique, sans succès [3]. Zavoïskii et ses collègues utilisent la méthode dite du "courant de grille ", beaucoup plus sensible que la méthode calorimétrique de Gorter. À la place de l'eau pure, ils utilisent des solutions de sels paramagnétiques et un système d'écoulement, afin de raccourcir le temps de relaxation du proton. En juin 1941, ils observent des signaux de résonance magnétique nucléaire (RMN). Les premières expériences ne sont pas reproductibles (en raison d'un défaut d'homogénéité du champ magnétique, non identifié à l'époque). Ils décident donc de ne pas publier leurs résultats.

Leurs recherches sont interrompues en 1941 à l'entrée en guerre de l'URSS. Les difficultés économiques, la pénurie de 


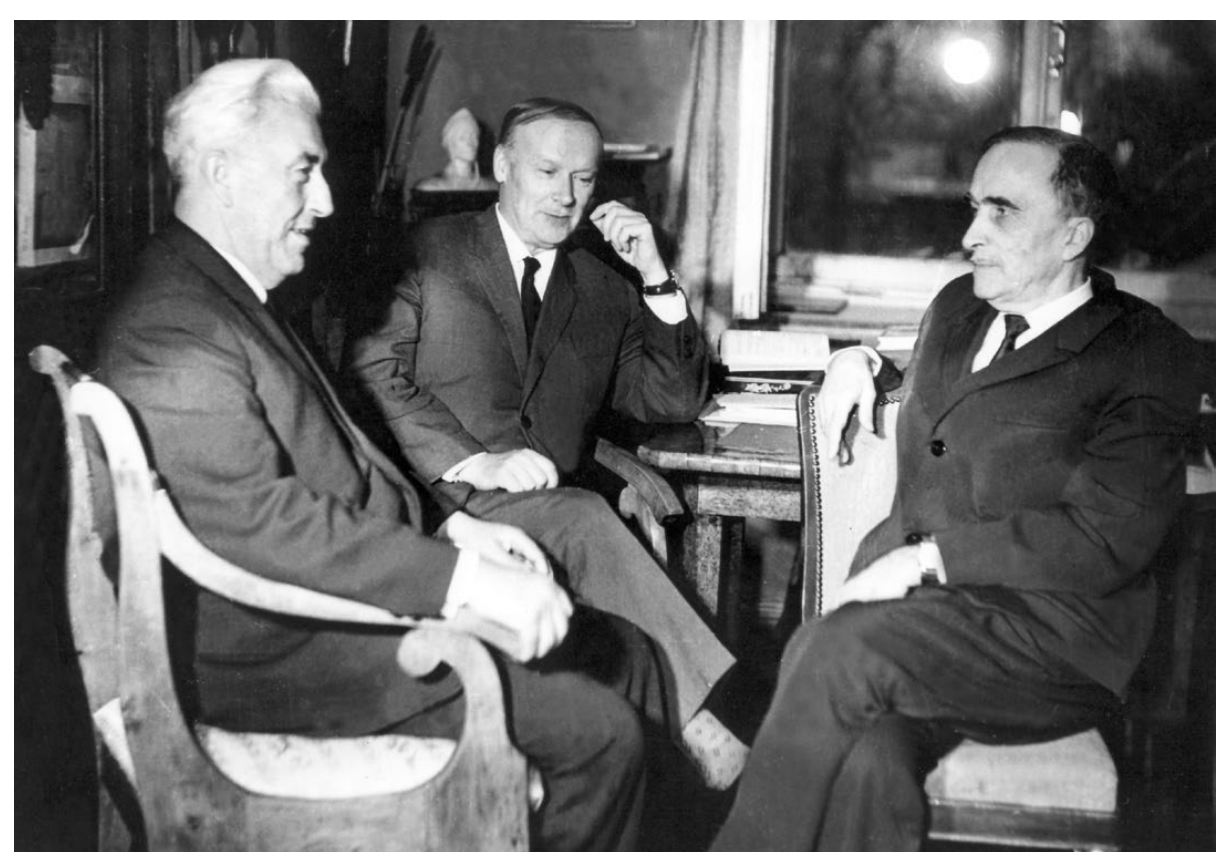

3. De gauche à droite : Semen Altshuler, Evguenii Zavoïskii et Boris Kozyrev à Kazan en 1968. (Photo : Mikhail L. Blatt.)

certains matériaux nécessaires aux expériences et l'absence de soutien des membres de l'Académie des sciences de l'Union Soviétique, ont pour conséquence le démantèlement du dispositif expérimental construit par Zavoïskii. Cette décision est prise par l'Académie des sciences sous prétexte que le montage expérimental est trop simple pour permettre la confirmation de la découverte scientifique du phénomène fondamental de la RMN. Plus tard, Semen Altshuler et Boris Kozyrev écrivirent : « Si Zavoïskii avait eu deux à trois mois pour poursuivre ses expériences, il aurait identifié la raison de la mauvaise reproductibilité de ses résultats et l'existence de l'absorption par résonance magnétique des protons aurait été prouvée. » [4]

\section{La découverte de la résonance paramagnétique électronique (RPE)}

En 1943, Zavoïskii retourne à ses recherches expérimentales à l'Université de Kazan. Cette fois, il entreprend d'étudier la relaxation paramagnétique électronique de sels paramagnétiques de manganèse et de cuivre dans des champs magnétiques perpendiculaires (voir encadré, p. 42). Le dispositif expérimental artisanal construit pour les expériences de RMN était tout à fait adapté à l'étude de la relaxation paramagnétique (voir la photo en haut de la page 39).

Zavoïskii conçoit et met en œuvre une méthode qui lui permet d'améliorer notablement la sensibilité de l'expérience. Il introduit une modulation du champ magnétique statique en ajoutant un champ magnétique alternatif parallèle au champ statique et ayant une fréquence beaucoup plus basse que celle du champ magnétique oscillant perpendiculaire au champ statique. En outre, le domaine des radiofréquences appliquées est étendu jusqu'à $100 \mathrm{MHz}$. Le 21 janvier 1944, Zavoïskii observe les pics d'absorption d'un champ d'ultrahaute fréquence dans des sels paramagnétiques soumis à un champ magnétique statique (fig. 4). Il découvre ainsi le phénomène de résonance paramagnétique électronique (RPE) en phase condensée et soumet un article pour publication le 12 juillet 1944 , date officielle de la découverte de la RPE [5].

Cette découverte résultait d'une part de ses recherches antérieures sur la résonance magnétique nucléaire, d'autre part de son extraordinaire intuition et de son approche originale des problèmes scientifiques. Il n'est pas surprenant que ses collègues l'aient appelé "le génie de l'expérience ". Dans ses expériences de RPE, Zavoïskii utilisait des ondes dans la gamme des mégahertz. Après la Seconde Guerre mondiale, des générateurs de fréquences de l'ordre du gigahertz sont disponibles et permettent d'améliorer la sensibilité de la détection du signal de RPE.

Bien que le physicien britannique Brebis Bleaney ait parfois été crédité de la découverte de la résonance paramagnétique électronique, sa première publication sur la RPE [6] fut envoyée le 19 décembre 1947, plus de trois ans après celle de Zavoïskii.

De 1945 à 1947, les activités scientifiques extrêmement fructueuses de Zavö̈skii sont à l'origine de nombreux projets reposant sur sa découverte de la RPE : il démontre expérimentalement les effets de l'antiferromagnétisme dans les phénomènes de résonance et découvre le phénomène de la résonance ferromagnétique. En 1948, il avait publié 18 articles sur la résonance et la relaxation paramagnétiques. Le livre de Boris Kochelaev et Youri Yablokov [7] offre une description très intéressante de l'histoire de l'Université de Kazan et des informations détaillées sur les premières recherches relatives à la résonance paramagnétique.

\section{Plusieurs autres contributions scientifiques importantes}

En 1947, Igor V. Kourtchatov invite Zavö̈skii à le rejoindre à Moscou et à participer au projet atomique soviétique à Sarov (Arzamas 16 à l'époque de l'URSS) et aux recherches sur les processus très rapides de physique des rayonnements et de physique des plasmas [8]. Une méthode d'enregistrement de signaux lumineux extrêmement courts et faibles est développée selon les directives de Zavoïskii. À partir de cette époque, ses recherches et activités expérimentales ont porté sur un très vaste domaine : la détection des traces des particules ionisantes et ses applications; la polarisation des faisceaux de particules et les cibles polarisées ; la fusion thermonucléaire contrôlée et la physique des plasmas ; le chauffage turbulent, etc. Des transducteurs électro-optiques multicascade pouvant mesurer une durée de signal comprise entre $10^{-14}$ et $10^{-12}$ seconde sont développés. Ces transducteurs furent ensuite appliqués en physique des plasmas, physique nucléaire, technologie laser, à l'astronomie et à la biologie. 


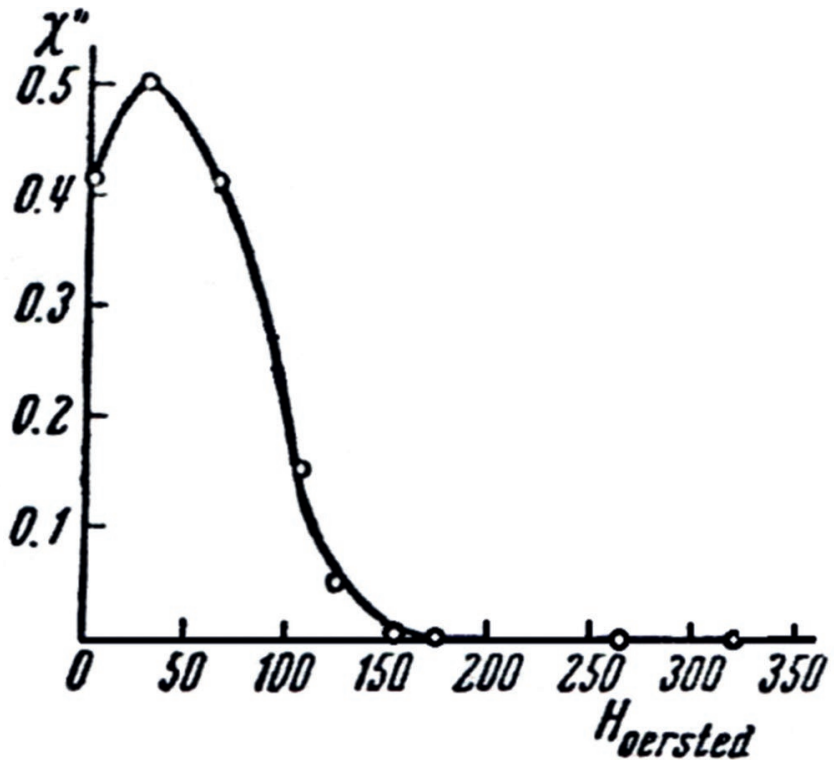

4. Le premier spectre de RPE obtenu pour une solution de chlorure de manganèse tétrahydraté $\left(\mathrm{MnCl}_{2} \cdot 4 \mathrm{H}_{2} \mathrm{O}\right)$ dans de l'alcool méthylique à la concentration de $0,175 \mathrm{~g} / \mathrm{cm}^{3}$, soumis à un champ magnétique statique $\mathrm{H}$ et à un champ magnétique oscillant perpendiculaire à $\mathrm{H}$, de longueur d'onde $25 \mathrm{~m}$ à $300 \mathrm{~K}$ [5]. On observe un maximum de la puissance radiofréquence absorbée quand la fréquence correspondant à la différence d'énergie $\Delta \mathrm{E}$ entre les niveaux de spin de l'ion de transition due à l'effet Zeeman est égale à celle du champ magnétique oscillant.

Zavoïskii développe une nouvelle méthode d'enregistrement des traces des particules ionisantes utilisant la chambre à traces de scintillation. Cette méthode est aussi le résultat du développement d'un dispositif original : un convertisseur à multichambre électro-optique sensible aux photons individuels. Cette technique extrêmement sensible d'enregistrement des images seuils ouvre de nouvelles possibilités pour les recherches en physique nucléaire, spectroscopie optique, astronomie, biologie, physique des plasmas et finalement en électronique quantique. En tant qu'authentique innovateur et grand enthousiaste des applications pratiques de ses résultats, Zavoïskii fait un grand travail de promotion de cette nouvelle technique auprès des physiciens, astronomes, biologistes et autres spécialistes. Il participe personnellement à la première utilisation expérimentale du convertisseur multichambre électro-optique sur un télescope à l'observatoire astrophysique de Crimée.

Zavoïskii conçoit une méthode de polarisation des faisceaux de particules chargées produites par des accélérateurs et participe à la conception de cibles polarisées pour des expériences de physique nucléaire.
À partir de 1958, Zavoïskii travaille sur le programme de la fusion thermonucléaire contrôlée. Dans ce cadre, il est le premier à utiliser la méthode électro-optique en spectrochronographie de plasmas. Avec ses collègues, il découvre le phénomène de la résonance magnéto-acoustique dans un plasma [9]. Il concentre ses efforts sur la recherche des mécanismes de dissipation de l'énergie dans le plasma, qui sont plus efficaces que les collisions de paires. Puis vient la découverte de la résistance anormalement élevée d'un plasma aux densités de courant élevées et le chauffage efficace de ce plasma, qui est à l'origine du développement rapide de recherches expérimentales et théoriques sur les interactions collectives et les processus non linéaires dans les plasmas [10,11]. Le phénomène du chauffage turbulent eut une importance significative dans le développement de la théorie des plasmas. Il est utilisé, en particulier, dans les méthodes de chauffage du plasma sans collision, qui sont largement appliquées dans les tokamaks modernes pour la fusion thermonucléaire.

Zavoïskii est le premier à indiquer la possibilité de chauffer un plasma dense jusqu'aux températures nécessaires à la fusion thermonucléaire avec des faisceaux d'électrons relativistes [12]. Cette sugges- tion est à l'origine d'une conception radicalement nouvelle d'un réacteur de fusion thermonucléaire contrôlée. La mise en œuvre de cette idée est devenue l'une des pierres angulaires du programme de recherche sur la fusion en URSS et à l'étranger.

En 1972, bien que gravement malade, Zavoïskii poursuit ses études théoriques, cherchant inlassablement de nouvelles pistes de recherche scientifique. Il consacre beaucoup de temps et d'efforts à son travail pour l'Académie des sciences de l'URSS en tant que membre de différents conseils scientifiques.

Le 9 octobre 1976, E.K. Zavoïskii meurt d'une attaque cardiaque.

\section{Une reconnaissance mondiale}

Découverte par E.K. Zavoïskii, la résonance paramagnétique électronique (RPE) fait partie des découvertes scientifiques significatives du XX ${ }^{\mathrm{e}}$ siècle. De 1959 à 1976, le nom de Zavoïskii a été proposé plusieurs fois pour le prix Nobel de physique et celui de chimie.

Les travaux scientifiques de E.K. Zavoïskii sont tenus en haute estime, et la reconnaissance de ses mérites par le gouvernement de l'Union Soviétique se manifeste par l'attribution de nombreux prix et médailles. En 1957, il reçoit le prix le plus prestigieux de l'URSS (prix Lénine) pour sa découverte de la RPE.

En 1969, une conférence scientifique internationale commémora le $25^{\mathrm{e}}$ anniversaire de la découverte de la RPE à Kazan. Lors de cette conférence, le physicien français Alfred Kastler, lauréat du prix Nobel, déclare : "La Volga commence à partir d'un petit ruisseau, croît de plus en plus et se transforme finalement en un énorme fleuve, aussi plein que la mer. La même chose s'est produite avec la résonance paramagnétique. Elle a commencé avec une expérience modeste réalisée ici à l'Université de Kazan, il y a 25 ans. Dans les années qui ont suivi, elle s'est transformée en un énorme champ d'investigations et a entrainé des milliers d'expériences et de publications."

Les activités scientifiques pluridisciplinaires de E. Zavoïskii sont à l'origine d'une quantité de nouvelles informations et de données sur divers phénomènes physiques et sur les propriétés de la matière. Cependant, ce qui caractérise son travail 
Pour comprendre le phénomène de résonance paramagnétique électronique, prenons le cas simple d'un ensemble de "radicaux libres ", c'est-à-dire de molécules ayant chacune un électron " non apparié ", dispersées dans un ensemble beaucoup plus grand de molécules diamagnétiques (non porteuses de moments magnétiques permanents). À chacun de ces radicaux libres est associé un moment magnétique, qui peut être considéré comme isolé des autres (grâce aux molécules diamagnétiques). L'ensemble de ces radicaux libres a un comportement paramagnétique.

L'électron non apparié a un spin 1/2 et un moment magnétique associé $\mu=-\mathrm{g} \mu_{\mathrm{B}} \mathrm{S}$, où g désigne un facteur de décomposition des niveaux d'énergie électroniques appelé facteur de Landé, $\mu_{B}$ le magnéton de Bohr et $\mathrm{S}$ le spin électronique, dont la projection $m_{s}$ sur un axe peut valoir $+1 / 2$ ou $-1 / 2$. Pour un radical libre ou un électron, le facteur $\mathrm{g}$ a une valeur très proche de 2. L'échantillon est placé dans un champ magnétique homogène et statique d'intensité $B$. Aux deux valeurs $+1 / 2$ et $-1 / 2$ du spin correspondent deux valeurs de l'énergie $+1 / 2 g \mu_{B} B$ et $-1 / 2 g \mu_{B} B$. Cette décomposition d'un niveau d'énergie sous l'effet du champ magnétique est appelée " effet Zeeman " (fig. E1). La différence d'énergie entre ces deux niveaux vaut $\Delta E=g \mu_{B} B$. La théorie statistique de Boltzmann s'applique à l'ensemble des spins et indique que ceux ayant l'énergie basse sont très légèrement plus nombreux que ceux ayant l'énergie haute. Le rapport du nombre de spins $+1 / 2$ au nombre de spins $-1 / 2$ vaut $\exp \left(-g \mu_{B} B / k_{B} T\right)$, où $k_{B}$ désigne la constante de Boltzmann et $\mathrm{T}$ la température absolue. Si l'on soumet ce système à un second champ magnétique oscillant à la fréquence $v=\Delta \mathrm{E} / \mathrm{h}$ (où $\mathrm{h}$ désigne la constante de Planck) et perpendiculaire au champ magnétique statique $B$, des radicaux libres dont le spin vaut $+1 / 2$ passent à l'état $-1 / 2$ et inversement. Du bilan énergétique non nul des changements d'état des spins électroniques, il résulte une absorption d'énergie par l'échantillon contenant ces spins.

À une fréquence de 9 gigahertz, correspond une différence d'énergie $\Delta \mathrm{E}=5,9610^{-24}$ joule $\left(3,710^{-5} \mathrm{eV}\right)$. Pour des électrons libres ou des radicaux libres ( $g$ très voisin de 2 ), le champ magnétique statique permettant la résonance magnétique vaut environ 0,3230 tesla, et le rapport entre les nombres des spins $+1 / 2$ et $-1 / 2$ vaut environ $\exp \left(-1,7510^{-3}\right) \cong 0,99825$ à la température ambiante $\left(20^{\circ} \mathrm{C}\right)$. Ce nombre étant très proche de 1 , la proportion des spins électroniques subissant un changement d'état est donc faible. Dans les spectromètres modernes, le changement d'état de certains spins électroniques induit une absorption des ondes hyperfréquence dans une cavité résonnante, qui est détectée grâce à un circuit électronique et produit un " signal de résonance ". Ce signal peut être enregistré en fonction de la valeur du champ statique B

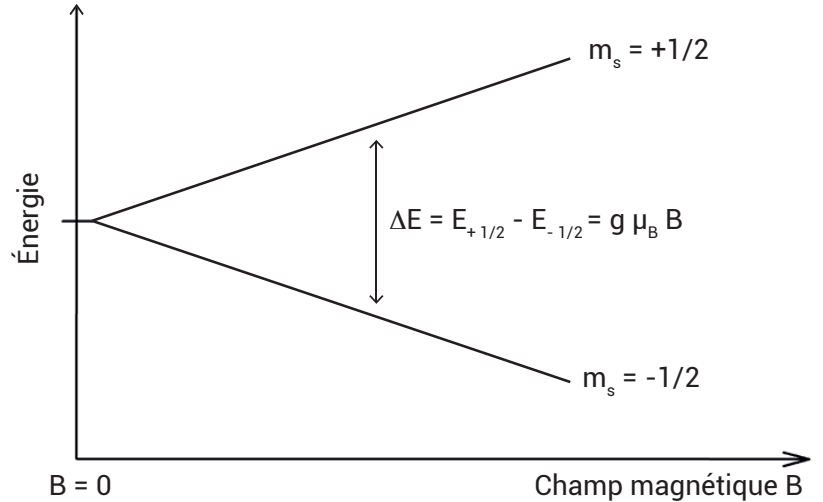

E1. Effet Zeeman et condition de résonance paramagnétique électronique.

appliqué. À son époque, Zavoïskii ne disposait pas des hyperfréquences (fréquences entre 1 et 100 gigahertz) et, pour améliorer la sensibilité de la détection de la résonance, il avait eu l'idée de moduler le champ magnétique statique $\mathbf{B}$ à une fréquence beaucoup plus basse que la fréquence de résonance paramagnétique électronique et de détecter le signal à la fréquence de modulation. Ce procédé lui permit de réduire considérablement le « bruit électronique » affectant le signal détecté, et ainsi d'améliorer la sensibilité de la chaine de détection du signal et de mettre en évidence la résonance paramagnétique électronique. Depuis cette époque, les spectromètres de RPE utilisent la modulation du champ statique à une fréquence pouvant être de 100 kilohertz et une détection synchrone.

L'application d'un champ magnétique oscillant à la fréquence $v=g \mu_{B} B / h$ à un échantillon contenant des radicaux libres a pour effet de tendre à égaliser les nombres de spins valant $+1 / 2$ et $-1 / 2$. À l'inverse, des processus de relaxation tendent à ramener ces nombres vers leurs valeurs à l'état d'équilibre de Boltzmann. Ces processus de relaxation sont non radiatifs et résultent du couplage entre les spins électroniques et les nombreux degrés de liberté des molécules impliquées. Pour que la résonance paramagnétique électronique soit détectée, il faut naturellement que les phénomènes de relaxation ne soient pas trop rapides. Pour des systèmes paramagnétiques tels que certains ions de métaux de transition ou de terres rares, le phénomène de résonance paramagnétique électronique ne peut être observé qu'à basse (voire très basse) température, lorsque les processus de relaxation sont suffisamment ralentis. 
c'est non seulement son désir d'obtenir ces informations, mais aussi celui de développer de nouvelles méthodes physiques efficaces de recherche. Il n'est donc pas étonnant que Zavoiskii ait insisté sur le fait que la résonance paramagnétique n'est pas seulement un phénomène, mais surtout une nouvelle technique expérimentale utilisée en physique du solide, chimie, sciences de la vie et géologie. La RPE permet, par exemple, de caractériser les centres paramagnétiques comportant un élément de transition, une terre rare ou de l'uranium, ou encore des radicaux organiques dans de nombreux matériaux tels que les verres, oxydes, minéraux naturels, matériaux d'électrodes pour batteries, etc. Elle est également utilisée pour étudier la structure des protéines, la cinétique des réactions chimiques, le transfert d'électrons ou le transfert de spin. De nombreux laboratoires à travers le monde utilisent la RPE, et la référence [13] témoigne de l'extraordinaire diversité des applications de cette spectroscopie.

En 1977, la médaille et le prix de la Société Internationale de Résonance Magnétique (ISMAR) sont décernés à titre posthume à E.K. Zavoïskii, témoignant de son énorme contribution à la découverte de la résonance paramagnétique.

Le laboratoire mémoriel Zavoïskii est ouvert en 1997 à l'Université d'État de Kazan, où Zavoïskii fit la première observation de signaux de résonance magnétique de spin. Parmi les pièces exposées se trouve un appareil de résonance magnétique en état de marche (photo en haut de la p. 39), décrit par Zavö̈skii dans ses notes de laboratoire.

E.K. Zavoïskii est considéré comme le fondateur de l'école de Kazan de la radiospectroscopie magnétique. Ses idées et suggestions pour de futurs projets scientifiques furent extrêmement fructueuses. En 1984, son nom fut donné à l'Institut Physico-Technique de Kazan (KPhTI).

Les auteurs adressent leurs remerciements cordiaux à l'académicien Kev M. Salikhov et au professeur Alexey A. Kalachev (Institut Physico-Technique Zavoïskii) pour de fructueuses discussions, des commentaires et suggestions sur la découverte et les applications de la RPE. Ils remercient Igor I. Silkin, directeur du musée Zavoïskii à Kazan, pour son aide à l'organisation d'une visite du musée et pour la transmission de photographies faisant partie des archives du musée.

\section{Références}

(*) Pour la recherche des articles de E. K. Zavoïskii publiés (traduits) en anglais, il faut utiliser deux transcriptions de son nom, « Zavoïskii E. » ou « Zavoisky E. ».

1• Е.К. Завойский, "Метод измерения потенциалов возбуждения атомов и молекул", Журнал Экспериментальной и Теоретической физики (ЖЭТФ), 6, n¹ (1936) 37-51. ("Méthode de mesure des potentiels d'excitation d'atomes et de molécules", Journal de Physique Expérimentale et Théorique, 1936).

2• Е.К. Завойский, Б.М. Козырев, “О влиянии постоянного магнитного поля на нагревание водных растворов электролитов в поле высокой частоты", Журнал Экспериментальной и Теоретической физики (ЖЭТФ), 6, n6, (1936) 612-614. ("Sur l'effet d'un champ magnétique constant sur le chauffage de solutions aqueuses d'électrolytes dans un champ haute fréquence ", Journal de Physique Expérimentale et Théorique, 1936).

3. C.J. Gorter, "Negative result of an attempt to detect nuclear magnetic spins", Physica III, 9 (novembre 1936) 995-998.

4• С.А. Альтшулер, Б. М. Козырев, в книге Чародей эксперимента: воспоминания об академике Е.К. Завойском, Москва, Наука (1994), стр. 14, ISBN 5-02-00819-2. (Le magicien de l'expérience : souvenirs sur l'académicien E.K. Zavoïskii, Moscou, 1994, p. 14).

5• E.K. Zavoïskii, "Paramagnetic relaxation of liquid solutions for perpendicular fields", J. Phys. Acad. Sci. URSS, 9, n³ (1945) 211-216.

6- B. Bleaney et B.P. Penrose, "Paramagnetic Resonance at Low Temperatures in Chromic Alum", Proc. Phys. Soc., 60 (1948) 395-396.

7• B.I. Kochelaev et Y.V. Yablokov, The beginning of paramagnetic resonance, World Scientific Publishing Co., Singapour (1995).

8• Voir l'article de Yu. N. Smirnov, "Evgueny Konstantinovich Zavoisky - A Participant of the Soviet Atomic Project", dans EPR Newsletter 17, 4 (2008), p. 7.

9• A. P. Akhmatov et al., "Magnetoacoustic Resonance in a Plasma", Soviet Physics JETP, 12 , $\mathrm{n}^{\circ} 3$ (mars 1961) 376-381.

10• M.V. Babykin et al., "New Results in Turbulent Heating in a Plasma", Soviet Physics JETP, $19, n^{\circ} 2$ (août 1964) 349-361.

11 •E. K. Zavoïskii et L. I. Rudakov, "Turbulent Heating in a Plasma" (traduction de Atomnaya Energiya, 23, n5 (novembre 1967) 417-431).

12• E.K. Zavoïsky, "Energetics in Fast Thermonuclear Processes", Sov. Phys. Usp., 15 (1973) 841

13. K. M. Salikhov, The Treasures of Eureka, Vol. 1. "Electron Paramagnetic Resonance: From Fundamental Research to Pioneering Applications \& Zavoïskii Award", New Zealand, 2009, AXAS Publishing Ltd.

\section{Le prix Zavoïskii}

En 1991, un prix Zavoïskii (International Zavoïskii Award) a été créé par l'Institut Physico-Technique Zavoïskii de Kazan, I'Université fédérale de Kazan, le gouvernement de la république du Tatarstan et l'éditeur Springer Verlag. Ce prix, d'un montant de 5000 euros, récompense une application ou un développement majeur de la RPE, dans tout domaine scientifique. II est reconnu par le Groupement AMPERE, la Société Internationale de RPE (EPR Society) et le présidium de l'Académie des sciences de Russie. 